OPEN ACCESS

Edited by:

Antonio Galvano,

University of Palermo, Italy

Reviewed by:

Feng Wei,

Tianjin Medical University Cancer Institute and Hospital, China

Jia Wei,

Nanjing Drum Tower Hospital, China

*Correspondence:

Yunpeng Liu

ypliu@cmu.edu.cn

Xiujuan Qu

xiujuanqu@yahoo.com

tThese authors have contributed equally to this work

Specialty section:

This article was submitted to Gastrointestinal Cancers,

a section of the journal

Frontiers in Oncology

Received: 26 August 2019 Accepted: 18 March 2020 Published: 22 April 2020

Citation:

Zhang M, Fan Y, Che X, Hou K, Zhang C, Li C, Wen T, Wang S, Cheng Y, Liu Y and Qu X (2020)

5-FU-Induced Upregulation of Exosomal PD-L1 Causes Immunosuppression in Advanced

Gastric Cancer Patients.

Front. Oncol. 10:492.

doi: 10.3389/fonc.2020.00492

\section{5-FU-Induced Upregulation of Exosomal PD-L1 Causes Immunosuppression in Advanced Gastric Cancer Patients}

\author{
Min Zhang 1,2†, Yibo Fan ${ }^{1,2 \dagger}$, Xiaofang Che ${ }^{1,2}$, Kezuo Hou ${ }^{1,2}$, Chaoxu Zhang ${ }^{1,2}, \mathrm{Ce} \mathrm{Li}^{1,2}$, \\ Ti Wen ${ }^{1,2}$, Shuo Wang ${ }^{1,2}$, Yu Cheng ${ }^{1,2}$, Yunpeng Liu ${ }^{1,2 *}$ and Xiujuan $Q u^{1,2 *}$ \\ ${ }^{1}$ Department of Medical Oncology, The First Affiliated Hospital of China Medical University, Shenyang, China, ${ }^{2}$ Key \\ Laboratory of Anticancer Drugs and Biotherapy of Liaoning Province, The First Affiliated Hospital of China Medical University, \\ Shenyang, China
}

Although the cytotoxic chemotherapeutic agent 5-fluorouracil (5-FU) is generally considered to directly kill cancer cells and exert immunostimulatory effects in advanced gastric cancer, accumulating evidence indicates that it upregulates the expression of PD-L1, a representative immune checkpoint blockade molecule involved in negative regulation of the immune response. It was reported that exosomes could transfer functional PD-L1 locally and distantly to suppress the antitumor immune response. However, whether 5-FU alters the expression of exosomal PD-L1 and induces immunosuppression in gastric cancer remains unclear. Herein, we found that 5-FU increased gastric cancer-derived exosomal PD-L1. Importantly, compared with baseline levels, circulating exosomal PD-L1 was significantly upregulated in 21 stage III-IV gastric cancer patients after two, four, and six repeated cycles of fluoropyrimidine treatment $(P=0.009, P=0.047$, and $P=0.023$, respectively), accompanied by decreased amounts of IFN- $\gamma$, TNF- $\alpha, \mathrm{IL}-2, \mathrm{IL}-6$, and GM-CSF $(P=0.014, P=0.004, P=0.009$, $P=0.031$, and $P=0.014$, respectively). Additionally, circulating exosomal PD-L1 was increased more significantly in clinical non-responders compared with responders $(P=0.018)$. Furthermore, exosomal PD-L1 induced apoptosis in Jurkat $T$ cells and inhibited $T$ cell activation in PBMCs, which could be partly reversed by nivolumab. These results suggested that 5-FU-induced upregulation of exosomal PD-L1 causes systemic immunosuppression in advanced gastric cancer following multiple cycles of chemotherapy, especially after two cycles.

Keywords: exosomal PD-L1, 5-fluorouracil, gastric cancer, immunosuppression, nivolumab

\section{INTRODUCTION}

Gastric cancer (GC) is the fifth most prevalent cancer and the third leading cause of cancer-related death worldwide. In 2018, GC caused 782,685 deaths (1). The majority of GC patients show advanced to late-stage disease at the time of diagnosis because efficient early diagnostic markers are unavailable (2). Chemotherapy, one of the main treatment methods, has been extensively applied in advanced gastric cancer. Accumulating evidence indicates that, although conventional chemotherapeutic drugs exert direct cytostatic/cytotoxic effects (3), they could alter tumor-reactive 
immune responses and even display immunosuppressive features (4), which may affect the survival of patients. 5-FU is the backbone substance of advanced gastric cancer chemotherapy (5). 5-FU kills tumor cells through misincorporation of fluoronucleotides into DNA and RNA molecules and by inhibition of the nucleotide synthesis enzyme thymidylate synthase (TS) (6). 5-FU was shown to selectively eliminate tumor-associated MDSCs resulting in enhanced T-celldependent antitumor immunity in several types of cancer (7). Nevertheless, a better understanding of the mechanism by which 5-FU may alter antitumor immune responses in gastric cancer patients, especially immune status changes after different cycles of 5-FU treatment, is important for the improvement of treatment efficacy.

Substantial evidence suggests that some chemotherapeutic drugs exert immunogenic effects that could improve the antitumor response $(3,8)$ by the following: $(1)$ promoting antigen presentation and recognition; (2) provoking immunogenic cell death (ICD) $(9,10)$; (3) increasing the amount of effector $\mathrm{T}$ cells; (4) reducing immunosuppressive cells, including MDSCs, regulatory T cells, and type II macrophages; and (5) regulating the secretion of cytokines. In recent years, the immunosuppressive effects of some chemotherapeutic drugs have been noticed, while much attention has been paid to the immunostimulatory aspect of chemotherapy (10). Several studies have suggested that chemotherapeutic drugs could impair antitumor immunity mainly in the following aspects: (1) reducing circulating immune cells, (2) inducing immunosuppressive monocytic myeloid cells (10), (3) upregulating PD-L1 to induce T cells anergy and immune evasion by interacting with PD-1 on activated $\mathrm{T}$ cells $(11,12)$, and (4) triggering chemotherapy-elicited extracellular vesicles (EVs) to exert prometastatic effects (13). Interestingly, it was reported that the doses and times of chemotherapeutic drugs as well as the methods of administration in vivo or in vitro might alter the immune system differently (14). Two studies have shown that 5-FU increases PD-L1 levels in breast and colorectal cancer cells, but little is known about PD-L1 level changes associated with 5-FU in gastric cancer. Moreover, whether 5FU concentration and treatment time affect PD-L1 in tumor cells and the immune system in advanced gastric cancer remains largely unknown.

Exosomes represent membrane-bound organelles containing various bioactive molecules and play a key role in intercellular communication, affecting physiological functions; in addition, they can "package" DNA, RNA, and proteins of tumor cells to the local or systemic body, facilitating tumor progression and

Abbreviations: 5-FU, 5-fluorouracil; GC, gastric cancer; TS, thymidylate synthase; ICD, immunogenic cell death; PD-L1, programmed death-ligand 1; PD-1, programmed death-1; EVs, extracellular vesicles; IL-2, interleukin-2; IL-4, interleukin-4; IL-6, interleukin-6; IL-8, interleukin-8; IL-10, interleukin-10; GMCSF, granulocyte-macrophage colony stimulating factor; TNF- $\alpha$, tumor necrosis factor- $\alpha$; IFN- $\gamma$, interferon- $\gamma$; ORR, objective response rate; PR, partial response; $\mathrm{SD}$, stable disease; $\mathrm{PD}$, progressive disease; PBMCs, peripheral blood mononuclear cells; PMA, phorbol 12-myristate 13-acetate; TCGA, The Cancer Genome Atlas; STAT4, signal transducer and activator of transcription 4; STAT5A, signal transducer and activator of transcription 5A; siRNA, small interfering RNA; shRNA, short hairpin RNA; NC, negative control. metastasis (15-17). Besides membrane-bound and extracellular soluble forms, several studies have demonstrated that PD-L1 also has exosomal forms (18-20). In addition, Mauro et al. confirmed that tumor-derived exosomes presenting PD-L1 could migrate to the draining lymph node, inducing local and/or systemic immunosuppression that promotes tumor progression (20). Our previous study revealed that exosomal PD-L1 is stable and might have stronger immunosuppressive activity than other forms of PD-L1 (21). Thus, we hypothesized that 5-FU may not only change PD-L1 expression in gastric cancer but also alter tumorderived exosomal PD-L1, exerting stronger and more extensive immunosuppressive effects.

In the present study, we investigated the effect of 5-FU on exosomal PD-L1 in patients with advanced gastric cancer. Clinical blood samples at baseline and after different cycles of treatment with fluoropyrimidine were used to assess the effects of fluoropyrimidine on circulating exosomal PD-L1 and the immune status. Compared with baseline levels, circulating exosomal PD-L1 was upregulated after two, four, and six cycles of fluoropyrimidine treatment $(P=0.009, P=0.047$, and $P=0.023$, respectively). Cytokines including interferon$\gamma$ (IFN- $\gamma$ ), tumor necrosis factor- $\alpha$ (TNF- $\alpha)$, interleukin (IL)2 , IL-6, and granulocyte-macrophage colony stimulating factor (GM-CSF) were decreased obviously following repeated cycles chemotherapy, especially after two cycles $(P=0.014, P=0.004$, $P=0.009, P=0.031$, and $P=0.014$, respectively). Further exploration demonstrated that tumor-derived exosomal PD-L1 induced apoptosis in Jurkat T cells and inhibited the activation of $\mathrm{T}$ cells in peripheral blood mononuclear cells (PBMCs) in vitro.

\section{MATERIALS AND METHODS}

\section{Patients and Plasma Collection}

This retrospective study was conducted on stage III-IV gastric cancer patients $(N=21)$, who were followed up at the First Hospital of China Medical University from 2013 to 2018. Patients were enrolled into the cohort if they met the following criterion: only received fluoropyrimidine standardized monochemotherapy (including 5-FU, Capecitabine, S-1). The study was approved by the Ethics Committee of China Medical University, and all research were conducted in accordance with ethical principles. The clinical characteristics and details of all patients were retrieved from the Hospital Information System. Samples of peripheral blood were collected from the patients into separator tubes immediately before and after chemotherapy, which were subjected to centrifugation at 3,000 rpm for $20 \mathrm{~min}$ at $4^{\circ} \mathrm{C}$ to isolate plasma for preparing plasma-derived exosomes.

\section{Cell Culture}

Human gastric cell lines MGC803, SGC7901, and AGS were obtained from the Type Culture Collection of the Chinese Academy of Sciences (Shanghai, China). MKN74 was obtained from JCRB (Osaka, Japan). MKN45 and NCI-N87 cells were obtained from ATCC (Maryland, USA). Jurkat T cells were obtained from the American Type Culture Collection (ATCC, Rockville, MD, USA). All cells were maintained in Roswell Park Memorial Institute (RPMI)-1640 medium (Gibco, Gaithersburg, 
MD, USA) containing $10 \%$ heat-inactivated fetal bovine serum (FBS), penicillin (100 $\mathrm{U} \mathrm{ml}^{-1}$ ), and streptomycin (100 mg $\mathrm{ml}^{-1}$ ) in a humidified incubator with a mixture of $95 \%$ air and $5 \% \mathrm{CO}_{2}$ at $37^{\circ} \mathrm{C}$. Cells were passaged every 2-3 days when they were $80 \%$ confluent and were tested monthly for mycoplasma contamination.

\section{Exosomes Purification}

Exosomes were isolated from plasma samples of gastric cancer patients using Exosome Precipitation Solution (ExoQuick ${ }^{\mathrm{TM}}$, SBI) following the manufacturer's instructions. Exosomes from supernatants of gastric cells were isolated by ultracentrifugation as described previously (22). MKN74 cells were cultured in RPMI-1640 medium with exosome-deleted FBS (depleted of bovine exosomes by overnight centrifugation at 100,000 $\times \mathrm{g}$ at $4{ }^{\circ} \mathrm{C}$ ). We collected cultured conditioned medium after $48 \mathrm{~h}$ and centrifuged at $3,000 \times \mathrm{g}$ for $15 \mathrm{~min}$ to remove cellular debris and dead cells. Then, the supernatant was filtered through $0.22 \mu \mathrm{M}$ pore filter to eliminate larger extracellular vesicles. Exosomes were pelleted by ultracentrifugation at $100,000 \times \mathrm{g}$ for $70 \mathrm{~min}$ for differential centrifugation. The exosomes were washed with phosphate-buffered saline (PBS) and were centrifugated at $100,000 \times \mathrm{g}$ for $70 \mathrm{~min}$ once again, then resuspended in PBS and stored at $-80^{\circ} \mathrm{C}$. Concentration of exosomes was determined by protein quantification (Micro BCA Protein Assay Kit; Thermo Scientific, Waltham, MA, USA) according to the manufacturer's instructions.

\section{Transmission Electron Microscopy}

Exosomes derived from MKN74 were fixed in $4 \%$ paraformaldehyde and placed on a formvar carbon-containing grid, contrasted, and then embedded in a mixture of uranyl acetate and methylcellulose, followed by observation under a JEM-1200EX transmission electron microscope (JEOL, Tokyo, Japan). We captured images at 70,000 $\times$ amplification.

\section{NanoSight Measurements}

Analysis of absolute size distribution of isolated exosomes was detected by a NanoSight NS300 instrument (Malvern Instruments, Malvern, UK) following the manufacturer's protocol.

\section{Enzyme-Linked Immunosorbent Assay}

The expression of soluble PD-L1 in cultured cells and PD-L1 in plasma-derived exosomes were detected by enzyme-linked immunosorbent assay (ELISA) (DY156, R\&D), as recommended by the manufacturer's protocol. The optical density (OD) value of each well was measured under $450 \mathrm{~nm}$ by Bio-RAD iMark Microplate Reader (Bio-RAD Laboratories Inc., Kyoto Japan). The concentration of PD-L1 was quantitated by standard curve.

\section{Cytokine Measurement Analysis}

The expression of cytokine in the plasma of gastric cancer patients was profiled by multiplex bead assay because of the high sensitivity. The experiment was performed using Bio-Plex Pro Human Cytokine 8-plex Assay (M50000007A, Bio-Rad). The detected cytokines included IL-2, IL-4, IL-6, IL-8, IL-10, IFN$\gamma$, GM-CSF, and TNF- $\alpha$, the detection of which was conducted following the manufacturer's instruction. Bio-Plex suspension array system and Bio-Plex Manager software measured the concentration of cytokines.

\section{Development of PD-L1-Knockdown Cells}

Lentiviral particles with short hairpin RNAs (shRNAs) targeting human PD-L1 gene (PD-L1-KD group) or scrambled shRNA control (NC group) cotransfected MKN74 cells with viral packing plasmid and PolyFect Transfection reagent (OBiO Technology, Shanghai, China) were performed according to the manufacturer's instruction. ShRNA sequences for PD-L1 were as follows: 5'-CCAGCACACUGAGAAUCAA-3' (sense), 5'-UUGAUUCUCAGUGUGCUGG-3' (antisense). MKN74 cells $\left(2 \times 10^{3} /\right.$ well $)$ were cultured in a 96-well-plate overnight and then transfected at a multiplicity of infection (MOI) of 100 , respectively. After transfection for $48 \mathrm{~h}$, we used fluorescence microscopy to validate the infection efficiency $>90 \%$. PD-L1-KD cells were selected in the presence of $2 \mu \mathrm{g} / \mathrm{ml}$ puromycin (SigmaAldrich) for $48 \mathrm{~h}$, validated by Western blot. We harvest and cultured the MKN74 PD-L1-KD cells for exosomes preparation.

\section{Small Interfering RNA Transfections}

STAT5A and Cbl-b small interfering RNA (siRNA) and the negative control (NC) were obtained from GeneChem (Shanghai, China). The sequences were as follows: STAT5A, 5'-AUGGAUAUGUGAAACCACAttUGUGGUUUCACAUAU CCAUca-3' and Cbl-b, 5'-CCTGATGGGAGGAGTTATA-3'; NC, 5'-UUCUCCGAACGUGUCACGUttACGUGACACGUUC GGAGAAtt-3'. MKN74 or MGC803 cells $\left(1.5 \times 10^{5} /\right.$ well $)$ were respectively cultured in six-well-plates overnight and then were transfected with siRNA or NC using lipofectamine 2000 (Invitrogen, Carlsbad, CA, USA) according to the manufacturer's instructions, performed as previously described. After $48 \mathrm{~h}$ of incubation with the transfected mix, the gene-silencing efficiency was verified by Western blot.

\section{Western Blot}

The protein from cells and exosomes were detected by Western blot, performed as previously described (23). The target bands of proteins were detected with an enhanced chemiluminescence reagent (SuperSignal Western Pico Chemiluminescent Substrate, Pierce; Thermo Fisher Scientific, Inc.) and were scanned by the Electrophoresis Gel Imaging Analysis System (DNR BioImaging Systems, Neve Yamin, Israel) and analyzed by $\mathrm{NIH}$ Image J software. Antibodies against glyceraldehyde 3-phosphate dehydrogenase (GAPDH) (25778), STAT5A (1081), and Cblb (\#sc8006) were purchased from Santa Cruz Biotechnology (Santa Cruz, CA, USA). Antibodies specific to PD-L1 (13684S), phospho-STAT4 (5267S), phospho-STAT5 (9351S), and STAT4 (2653) were from Cell Signaling Technology (Danvers, MA, USA). Antibodies against CD9 (ab92726) and CD63 (ab193349) were from Abcam (Danvers, MA, USA).

\section{RNA Isolation and Reverse Transcription-Quantitative Real-Time PCR}

The isolation of total RNA was performed as previously described (23). For microRNAs, The One Step PrimeScript ${ }^{\circledR}$ 
miRNA cDNA Synthesis Kit (Takara, Japan) was used for RNA reverse transcription. The comparative cycle threshold (Ct) method was used to calculate relative expression of miR-940, and the expression of U6 small nuclear RNA was used as reference. The forward primer for miR-940 was $5^{\prime}$ AAGGTTTAGCCGCTCCCCAAA- $3^{\prime}$ and that for U6 internal control was forward $5^{\prime}$-GCTTCGGCAGCACATATACTAAAAT$3^{\prime}$ and reverse 5'-CGCTTCACGAATTTGCGTGTCAT-3', respectively. The Uni-miR qPCR Primer was included in the kit. SYBR $^{\circledR}$ Premix Ex Taq ${ }^{\mathrm{TM}}$ II (Perfect Real Time) (Takara, Japan) was used for monitoring the amount of microRNA (miRNA). The PCR conditions were $30 \mathrm{~s}$ at $95^{\circ} \mathrm{C}$, followed by 45 cycles at $95^{\circ} \mathrm{C}$ for $5 \mathrm{~s}$ and $58^{\circ} \mathrm{C}$ for $25 \mathrm{~s}$. The threshold cycle and $2^{-\Delta \Delta \mathrm{Ct}}$ method were used for calculating the relative amount of the target RNA.

\section{Flow Cytometry Analysis}

PD-L1 on the membrane of MKN74 and MGC803 was detected by flow cytometry. Briefly, cells were incubated with PE antihuman CD274 (557924, BD) for membrane staining for $20 \mathrm{~min}$ at $4^{\circ} \mathrm{C}$ and were washed with PBS. Acquisition of 10,000 events in the gated population was performed in BD Accuri C6. The expression of CD9 on the membrane of MKN74-derived exosomes was detected by flow cytometry. Briefly, exosomes were incubated with aldehyde/sulfate latex beads (A37304, Thermo Fisher Scientific) in PBS and then added with $1 \mathrm{M}$ glycine and $10 \%$ bovine serum albumin (BSA), respectively. Exosomes were incubated with PE antihuman CD9 (555372, BD) for membrane staining and were detected by $\mathrm{BD}$ Accuri C6. Isotype control Ab staining was used as a negative control.

\section{PBMCs Separation and Cell Culture}

PBMCs were obtained from healthy donors $(N=3)$ and were isolated from venous blood $(5 \mathrm{ml})$ by density gradient centrifugation (Allegra X-15R Centrifuge, Beckman Coulter). Briefly, blood diluted in PBS was layered over Ficoll-Paque (Sinopharm Chemical Reagent Co., Ltd, China) and centrifuged at $400 \times \mathrm{g}$ for $30 \mathrm{~min}$ at $25^{\circ} \mathrm{C}$. The mononuclear cell band was recovered and washed twice in PBS. After separation, PBMCs were maintained in RPMI-1640 medium containing 10\% heat-inactivated FBS, penicillin $\left(100 \mathrm{U} \mathrm{ml}^{-1}\right)$, and streptomycin $\left(100 \mathrm{mg} \mathrm{ml}^{-1}\right)$ in a humidified incubator with a mixture of $95 \%$ air and $5 \% \mathrm{CO}_{2}$ at $37^{\circ} \mathrm{C}$.

\section{Function Analysis}

Aliquots $\left(2 \times 10^{5}\right)$ of PBMCs were placed in wells of 96well-plates, and $\mathrm{T}$ cells in PBMCs were activated by human CD3/CD28 T cell activator (\#10971, Stemcell Technologies) for $6 \mathrm{~h}$ at $37^{\circ} \mathrm{C}$ and then coincubated with MKN74-derived NC exosomes or PD-L1-KD exosomes for $48 \mathrm{~h}$ in the presence or absence of nivolumab (Bristol-Myers Squibb Company, Princeton, NJ, USA). PBMCs were stained with the following antibodies: FITC antihuman CD3 (561806, BD), APC antihuman CD69 (560967, BD), and PE antihuman CD25 (560989, BD). Isotype control $\mathrm{Ab}$ staining was used as a negative control.

\section{Cell Apoptosis Assay}

Jurkat $\mathrm{T}$ cells $\left(3 \times 10^{5} /\right.$ well $)$ were activated with $50 \mathrm{ng} / \mathrm{ml}$ phorbol 12-myristate 13-acetate (PMA; Sigma-Aldrich) for $24 \mathrm{~h}$ and then were coincubated with MKN74-derived NC exosomes or PD-L1-KD exosomes $(200 \mu \mathrm{g} / \mathrm{ml})$ for $48 \mathrm{~h}$. Then, cells were harvested and stained using an Annexin Vfluorescein isothiocyanate/propidium iodide apoptosis detection kit (BMS500FI-100; Invitrogen; Thermo Fisher Scientific, Inc.) in the dark at room temperature. After being washed and resuspended in PBS, the samples were determined and analyzed by BD Accuri C6.

\section{Bioinformatic Analysis}

UCSC Genome Browser (http://www.genome.ucsc.edu/) predicted DNA sequences in promoter regions of PD-L1. Then, the Promoter 2.0 Prediction Server (http://www.cbs.dtu.dk/ services/Promoter/) predicted transcription factors that interact with sequences in promoter regions within a dissimilarity margin $\leq 15 \%$. Animal TFDB 3.0 (https://bioinfo.life.hust. edu.cn/Animal TFDB/\#!/) also predicted transcription factors according to the predicted DNA sequences, and there were 15 transcription factors in common. Correlation of mRNA-seq data of predicted transcription factors and PD-L1 was evaluated based on the TCGA-STAD RNA-Seq-HTSeq-FPKM database from The Cancer Genome Atlas (TCGA, https://tcga-data.nci. nih.gov/docs/publications/tcga/) and the gene expression profile of GSE62254 in GC from the NCBI-GEO database (http:// www.ncbi.nlm.nih.gov/geo/) by Pearson correlation coefficient, as appropriate.

\section{Data and Statistical Analysis}

The experimental results were reported as mean \pm standard deviation (SD), and the mean values were calculated from more than three independent experiments. SPSS 16.0 software (IBM, USA) was used for all statistical analyses. GraphPad-Prism version 6.0 (GraphPad Software, USA) was used to perform graphics. Comparisons between two groups were performed using the Student's $t$-test. Comparisons between multiple groups were performed using the one- or two-way ANOVA. $P<0.05$ indicated that there existed statistical differences $\left({ }^{*} P<0.05,{ }^{* *} P\right.$ $\left.<0.01,{ }^{* * *} P<0.001,{ }^{* * * *} P<0.0001\right)$.

\section{RESULTS}

\section{5-FU Upregulates PD-L1 in Human Gastric Cancer Cells}

To analyze PD-L1 expression in gastric cancer cell lines, Western blot was performed to measure PD-L1 levels in AGS, MKN45, MKN74, SGC7901, MGC803 and NCI-N87 cells. As shown in Figure 1A, MKN74 cells expressed high levels of PD-L1, while low levels of PD-L1 were detected in MGC803 cells. Since previous studies have demonstrated that chemotherapy treatment might increase PD-L1 expression in some cancer types $(11,24,25)$, we aimed to explore whether 5-FU has a similar effect in gastric cancer. The gastric cancer MKN74 and MGC803 cell lines, with high and low expression of PD-L1, respectively, were incubated with 5 -FU $(0,0.1,1.0 \mu \mathrm{g} / \mathrm{ml})$, and then, PD-L1 
A

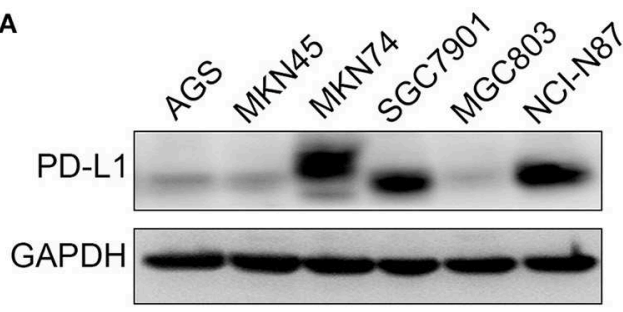

B

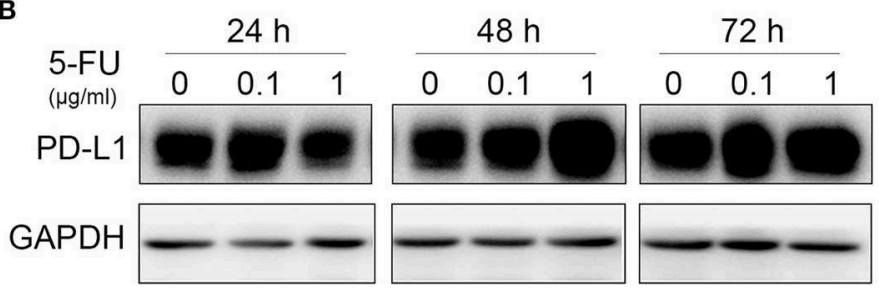

MKN74
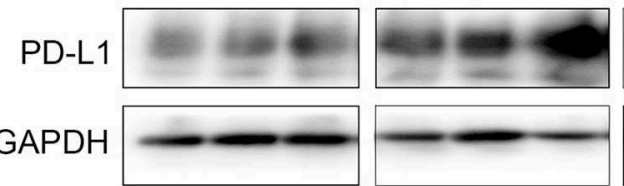

GAPDH

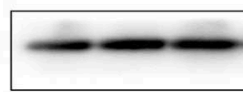

MGC803
D

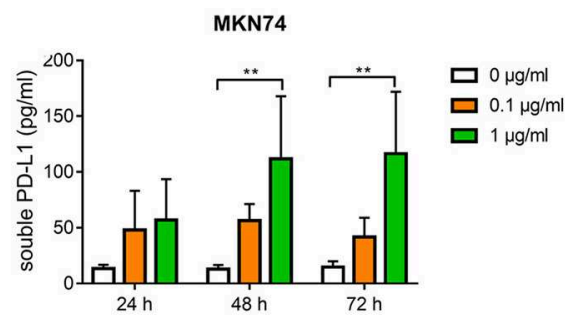

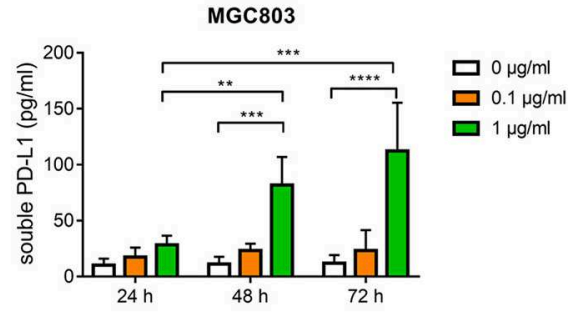

C
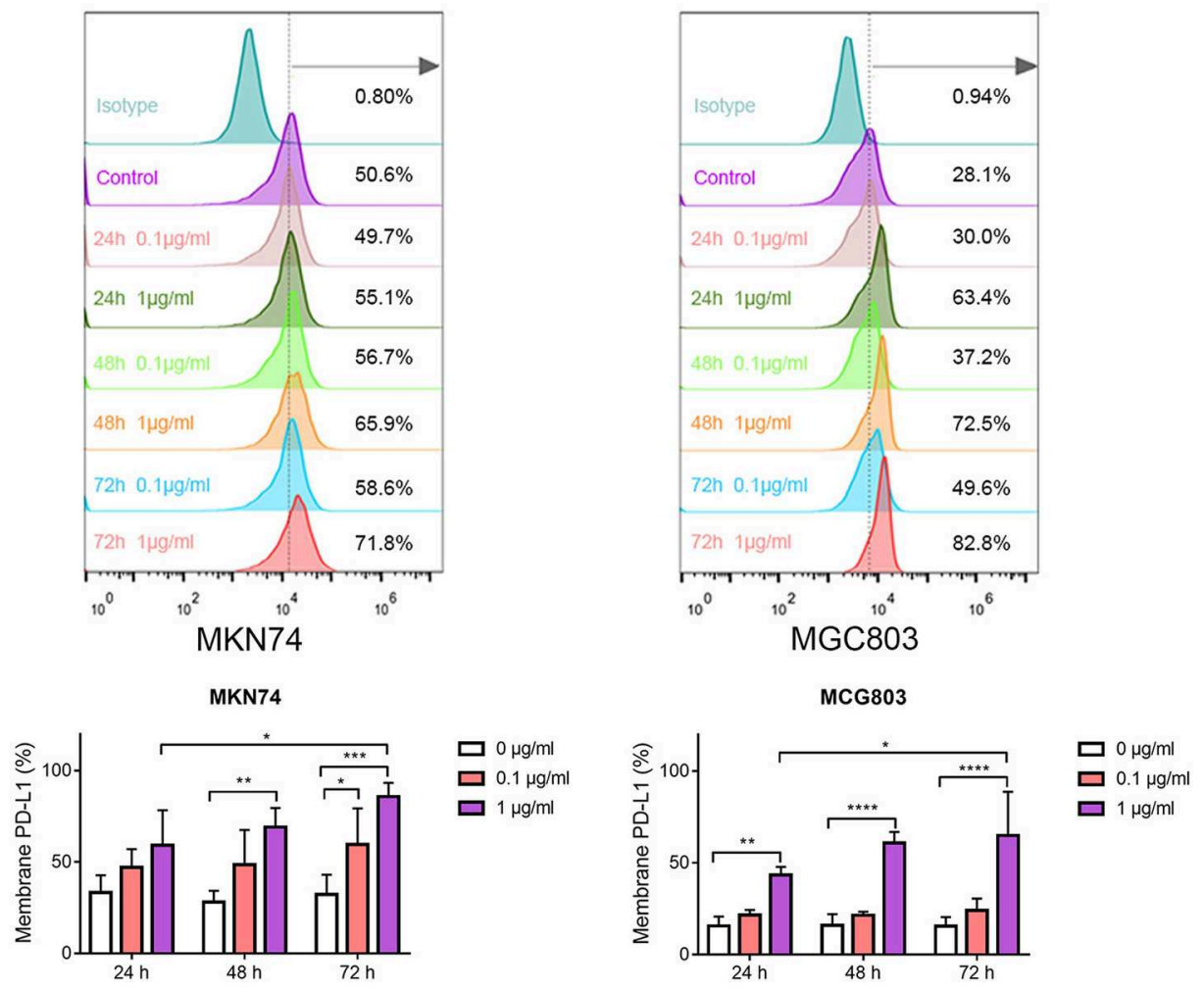

FIGURE 1 | 5-Fluorouracil (5-FU) upregulates programmed death-ligand 1 (PD-L1) in human gastric cancer cells. (A) Western blot was performed to measure PD-L1 levels in gastric cancer cell lines, including AGS, MKN45, MKN74, SGC7901, MGC803, and NCI-N87 cells. MKN74 and MGC803 cells were incubated with 5-FU (0, $0.1,1.0 \mu \mathrm{g} / \mathrm{ml}$ ) for 24, 48, and 72 h, respectively. (B) Western blot detected PD-L1 expression in MKN74 and MGC803 cells. (C) PD-L1 levels on the membrane of MKN74 and MGC803 cells were measured by flow cytometry. (D) Soluble PD-L1 levels in the cell culture supernatant were detected by ELISA. Data represent mean $\pm \mathrm{SD}, P<0.05$ indicates that there existed statistical differences $\left({ }^{\star} P<0.05,{ }^{\star \star} P<0.01,{ }^{\star \star \star} P<0.001,{ }^{\star \star \star \star} P<0.0001\right)$. Data were representative of three independent experiments. 
A 5-Fluorouracil

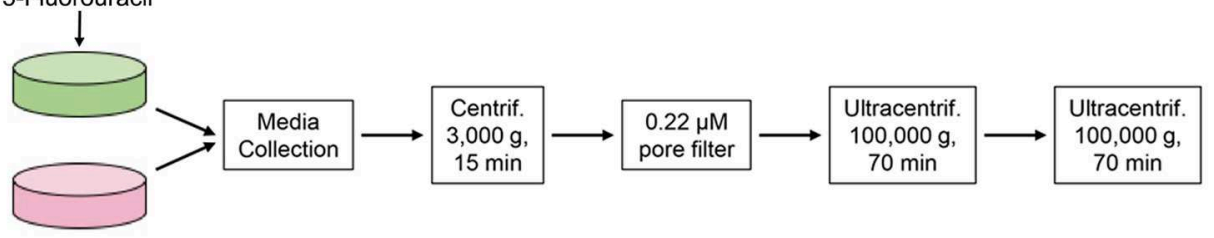

MKN74

B

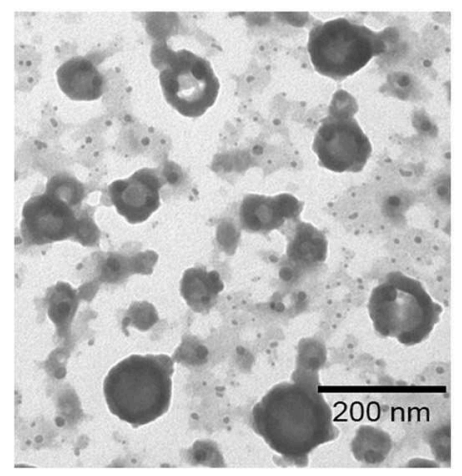

D

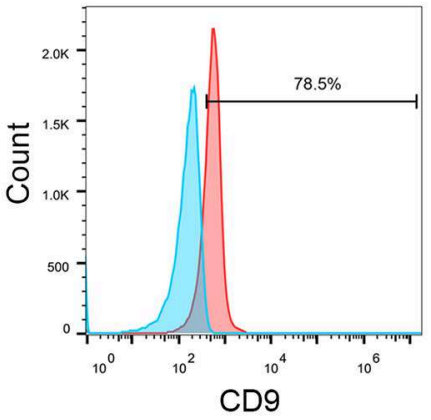

C
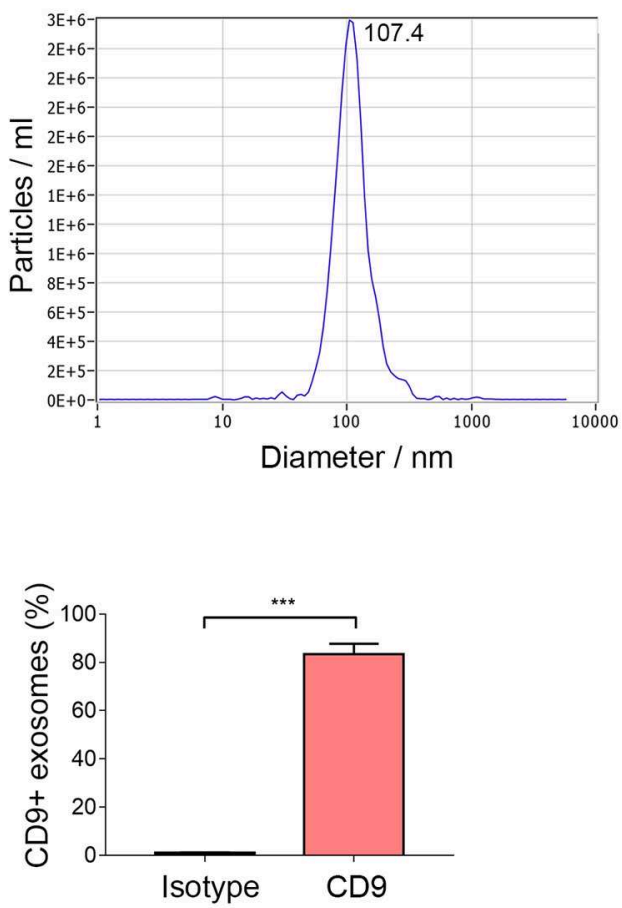

E

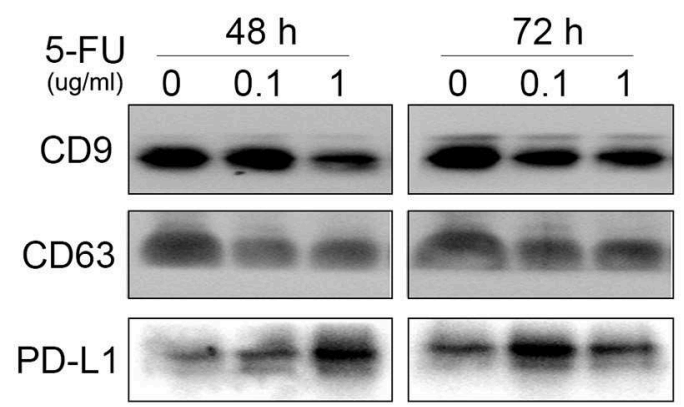

FIGURE 2 | 5-Fluorouracil (5-FU) increases the secretion of exosomal programmed death-ligand 1 (PD-L1). MKN74 cells were incubated with 5-FU (0, 0.1, 1.0 $\mu \mathrm{g} / \mathrm{ml})$ for 48 and $72 \mathrm{~h}$, respectively. (A) Schematic illustration of MKN74 cell-derived exosomes isolation and purification from the supernatants by ultracentrifugation. (B) MKN74-derived exosomes were validated by transmission electron microscopy (TEM). Scale bar: $200 \mathrm{~nm}$. (C) Size distribution of exosomes was analyzed by Nanosight. (D) CD9 levels on the surface of exosomes were detected by flow cytometry analysis. Data represent mean \pm SD, and Student's $t$-test was used to evaluate the statistical significance (** $P<0.001)$. (E) Western blot analysis of exosomes biomarker CD9 and CD63, and exosomal PD-L1 amounts. Equal amounts of proteins obtained from exosomes were immunoblotted with anti-CD9, anti-CD63, and anti-PD-L1 antibodies. 
level was detected at the 24,48 , and $72 \mathrm{~h}$ time points. Western blot showed that PD-L1 levels in both MKN74 and MGC803 cells were increased by $5-\mathrm{FU}$ in a dose- and time-dependent manner, with maximum value obtained after treatment with 5 -FU at $1.0 \mu \mathrm{g} / \mathrm{ml}$ for $72 \mathrm{~h}$; meanwhile, 5-FU-induced PD-L1 upregulation was more pronounced in MGC803 cells compared with MKN74 cells (Figure 1B). Additionally, 5-FU increased membrane expression of PD-L1 in MKN74 and MGC803 cells, in a dose- and time-dependent manner, as detected by flow cytometry (Figure 1C). It was reported that besides membranebound forms, PD-L1 also has soluble forms (26-28). 5-FU also increased the amounts of soluble PD-L1 in cell culture supernatants, as detected by ELISA (Figure 1D). Taken together, these results showed that 5-FU upregulated PD-L1 in a dose- and time-dependent manner in gastric cancer cell lines.

\section{5-FU Increases the Secretion of Exosomal PD-L1}

Accumulating evidence indicates that various cancer cells secrete exosomal PD-L1, including breast cancer, melanoma, prostate cancer, and glioblastoma (18-20, 29), which might play a crucial role in tumor progression. Our previous study showed that human gastric cancer cells also produce exosomal PD-L1 (21). However, whether 5-FU alters exosomal PD-L1 expression is unclear. To address this, we incubated MKN74 cells with 5 -FU $(0,0.1,1.0 \mu \mathrm{g} / \mathrm{ml})$ for 48 and $72 \mathrm{~h}$, respectively, and isolated MKN74-cell-derived exosomes from the supernatant by ultracentrifugation (Figure 2A). Exosomes confirmed by transmission electron microscopy (TEM) were limited by bilayer membranes with a characteristic ovoid or round shape (Figure 2B). NanoSight measurement showed that extracellular vesicles were $\sim 107.4 \mathrm{~nm}$ in diameter (Figure 2C). A large proportion of exosomes were positive for the biomarker CD9, as detected by flow cytometry analysis (Figure 2D). Next, PDL1 amounts in exosomes were examined in samples with similar levels of the exosome biomarkers CD9 and CD63. Exosomal PD-L1 levels were increased significantly after 5-FU treatment, with maximum value after treatment at $1.0 \mu \mathrm{g} / \mathrm{ml} 5$-FU for $48 \mathrm{~h}$ and $0.1 \mu \mathrm{g} / \mathrm{ml}$ for $72 \mathrm{~h}$ (Figure 2E), indicating that 5 -FU caused gastric cancer cells to produce exosomal PD-L1.

\section{Effects of Fluoropyrimidine on Circulating Exosomal PD-L1 and the Immune Status in Gastric Cancer Patients}

Previous studies assessing the effects of $5-\mathrm{FU}$ on PD-L1 expression are limited to mouse models. The effects of 5FU on exosomal PD-L1 have been neglected, while much attention has been paid to the membrane form. It is essential to evaluate the change in circulating exosomal PD-L1 levels following multiple cycles of 5-FU treatment in advanced gastric cancer patients. We obtained matched blood samples at baseline and after different cycles of chemotherapy from 21 stage IIIIV gastric cancer patients, who only received fluoropyrimidine standardized chemotherapy (including 5-FU, Capecitabine or S-1) (Figure 3A). Compared with baseline levels, circulating exosomal PD-L1 was upregulated after two $(N=21, P=$
$0.009)$ and four $(N=10, P=0.047)$ cycles of fluoropyrimidine treatment (Figure 3B). There were only three patients with blood samples after six cycles of chemotherapy, who showed upregulated circulating exosomal PD-L1 $(N=3, P=0.023)$ (Figure S1A).

To further investigate the immune status after different cycles of chemotherapy, we analyzed the absolute counts of $\mathrm{T}$ cell subgroups and the levels of cytokines in gastric cancer patients. The absolute counts of $\mathrm{CD}^{+}$and $\mathrm{CD}^{+}{ }^{+} \mathrm{T}$ cells showed a reduction trend but without statistical significance $(N=8 ; P$ $=0.4023$ and $P=0.4893$, respectively) (Figure 3C). As shown in Figure 3D, IFN- $\gamma$, TNF- $\alpha$, IL-2, IL-6, and GM-CSF amounts were decreased significantly after two cycles of treatment $(P$ $=0.014, P=0.004, P=0.009, P=0.031$, and $P=0.014$, respectively), but all were recovered at different degrees after four and six cycles of treatment (Figure S1B). The changes in exosomal PD-L1 was not associated with any changes of all eight immune cytokines in the clinical blood samples after two and four cycles of treatment $(P>0.05)$ (Tables S1, S2). The sample size of patients with six cycle treatments $(N=3)$ was too small to conduct any correlation analysis. These results suggested that repeated cycles of fluoropyrimidine treatment not only increased circulating exosomal PD-L1 amounts but also impaired the immune status and functions by inhibiting cytotoxic cytokines.

Next, we determined whether the change of circulating exosomal PD-L1 levels was associated with the treatment efficacy of fluoropyrimidine. Objective response rate (ORR) is an important indicator for evaluating the effectiveness of antitumor drugs. Based on that, among 21 gastric cancer patients, those showing treatment efficacy as complete response (CR), or PR were considered responders. Meanwhile, patients whose treatment efficacy was SD or PD were considered nonresponders. We observed that circulating exosomal PD-L1 amounts were elevated obviously in non-responders while only slightly increasing or even decreasing in responders $(P=0.018)$. Meanwhile, the absolute counts of $\mathrm{CD}^{+}$and $\mathrm{CD} 8^{+} \mathrm{T}$ cells were decreased more significantly in non-responders compared with responders, although no statistically significant differences were observed ( $P=0.1025$ and $P=0.6689$, respectively) (Figure 3E). Additionally, the changes in cytokines showed no differences between responders and non-responders (Figure S1C).

\section{Exosomal PD-L1 Induces Jurkat T Cell Apoptosis and Suppresses T Cell Activation in PBMCs}

We aimed to assess whether exosomal PD-L1 derived from human gastric cancer has suppressive effects on $\mathrm{T}$ cell function, similar to PD-L1 found in the membrane of tumor cells. The expression of PD-L1 was obviously lower in PD-L1-KD MKN74 cells compared with that in PD-L1-NC MKN74 cells (Figure 4A). Jurkat $\mathrm{T}$ cells were activated by $50 \mathrm{ng} / \mathrm{ml}$ PMA, as verified by significant upregulation of CD69 on the cell surface (Figure 4B). As shown in Figure 4C, apoptosis in activated Jurkat $\mathrm{T}$ cells was enhanced significantly after exposure to exosomes presenting PD-L1 compared with PD-L1-KD exosomes. Given its ability to induce $\mathrm{T}$ cell apoptosis, whether exosomal PD-L1 inhibits 
A Gastric cancer patients $(\mathrm{N}=21)$

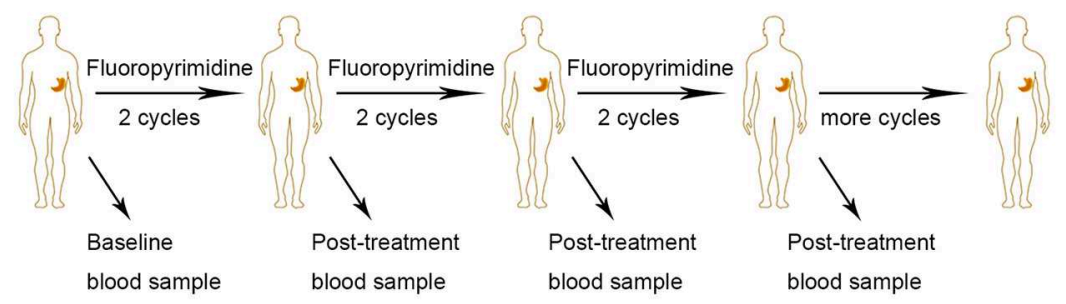

B

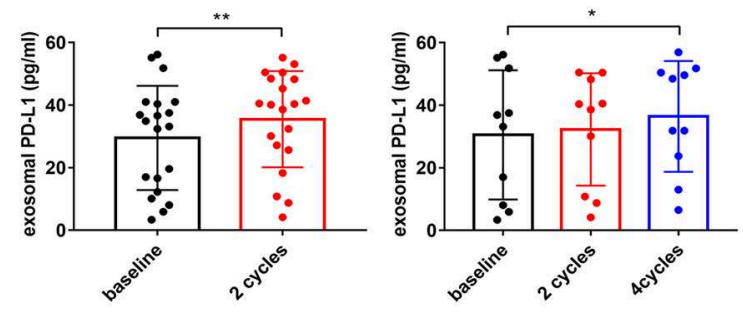

C

CD4+ T cell

CD8+ T cell
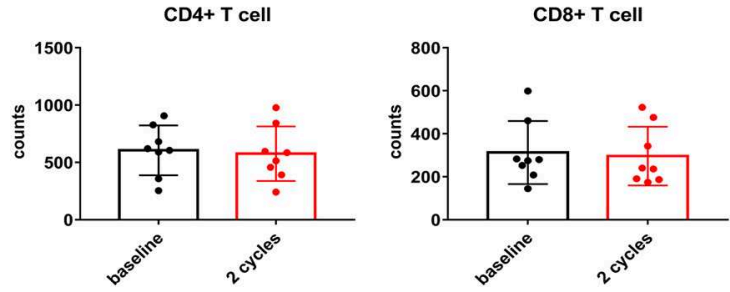

D
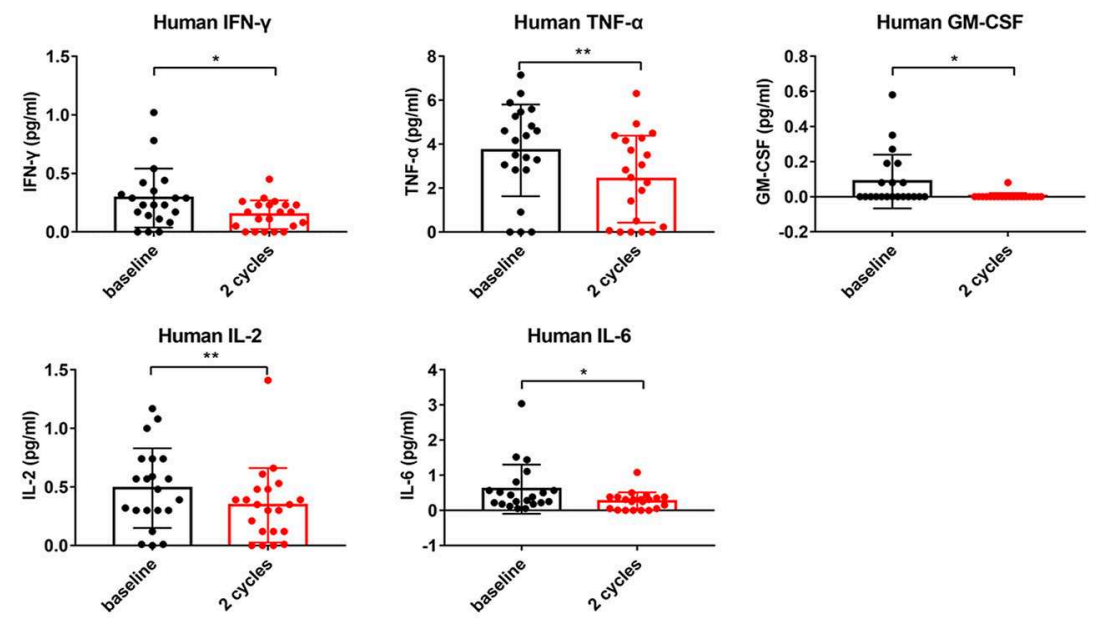

E
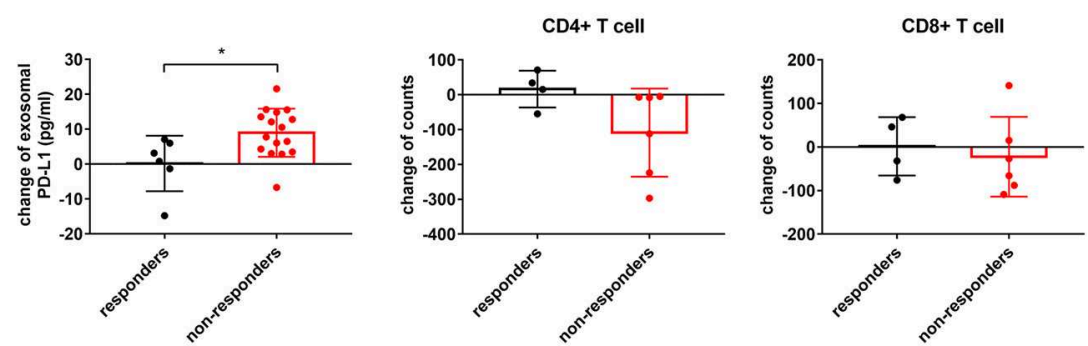

FIGURE 3 | Fluoropyrimidine increases circulating exosomal programmed death-ligand 1 (PD-L1) in gastric cancer patients. (A) Schematic illustration of blood samples collection at baseline and after two, four, and six repeated cycles of fluoropyrimidine chemotherapy treatment from stage III-IV gastric cancer patients 
FIGURE 3 | ( $N=21)$. (B) Plot of circulating exosomal PD-L1 levels in gastric cancer patients at baseline and after two cycles $(N=21$, one outlier was deleted) and four cycles $(N=10)$ of fluoropyrimidine chemotherapy treatment. (C) Left: Plot of the absolute counts of CD4 ${ }^{+} \mathrm{T}$ cells. Right: Plot of the absolute counts of CD8 ${ }^{+} \mathrm{T}$ cells $(N=8)$. (D) The levels of interferon- $\gamma(\mathrm{IFN}-\gamma)$, tumor necrosis factor- $\alpha$ (TNF- $\alpha$ ), interleukin (IL)-2, IL-6, and granulocyte-macrophage colony stimulating factor (GM-CSF) in plasma were analyzed by multiplex bead assay at baseline and after two cycles of fluoropyrimidine chemotherapy. (E) Plot of changes of circulating exosomal PD-L1 levels and the absolute counts of T cells in responders and non-responders. The two-tailed paired $t$-test was used in statistical analysis where appropriate to evaluate the statistical significance $\left({ }^{\star} P<0.05,{ }^{\star \star} P<0.01\right)$.

$\mathrm{T}$ cell activation in PBMCs was assessed. After exposure to exosomes derived from the NC group, cells expressing CD69, an "early" activation marker, showed a more pronounced percentage decrease (Figure 4D). In addition, the fraction of cells positive for CD25, a "late" activation marker, also showed a decreasing trend, although statistical significance was not achieved (Figure 4E). To ensure that the above observations were specific to PD-L1, we next included the anti-PD1 antibody nivolumab $(0.1 \mu \mathrm{g} / \mathrm{ml})$ in the assays to block PD-1 signaling after T cell exposure to NC exosomes. As expected, CD69 and CD25 amounts on T cells were increased to levels comparable to those obtained after exposure to PD-L1-KD exosomes (Figures 4D,E). Taken together, these data showed that exosomal PD-L1 in human gastric cancer had a direct effect in inhibiting the functions of T cells, which could be attenuated by nivolumab.

\section{MiR-940/Cbl-b/STAT5A Are Involved in 5-FU-Induced Upregulation of PD-L1 in Gastric Cancer Cells}

Through the online database UCSC Genome Browser, we predicted sequences in the promoter region of PD-L1. Promoter 2.0 Prediction Server and Animal TFDB 3.0 were utilized to predict transcription factors according to the promoter sequences and 15 transcriptional factors were mutually identified to possibly participate in $\mathrm{PD}-\mathrm{L} 1$ regulation (Figure 5A). We performed correlation analysis between these transcription factors and PD-L1. Results with TCGA-STAD dataset showed that five transcriptional factors were associated with PD-L1, including FOXP3 $(r=0.3003, P<0.0001)$, STAT4 $(r=0.3629, P$ $<0.0001)$, STAT5A $(r=0.4252, P<0.0001)$, TBP $(r=0.1223, P$ $=0.0181)$, and SP1 $(r=0.2902, P<0.0001)$ (Figure 5B). Results with GSE62254 dataset in gastric cancer in the NCBI-GEO database showed that seven predicted transcriptional factors were associated with PD-L1, including STAT4 $(r=0.577, P<$ $0.0001)$, STAT5A $(r=0.451, P<0.0001)$, YY1 $(r=-0.166, P$ $=0.008)$, POU2F1 $(r=-0.165, P=0.008)$, AR $(r=-0.152$, $P=0.015)$, RELA $(r=0.206, P=0.001)$, SP1 $(r=0.155, P$ $=0.013$ ) (Figure 5C). We made an intersection between the two cohorts and identified three transcriptional factors of PDL1, including SP1, STAT4, and STAT5A (Figure 5D). Based on their high correlation coefficients $(r>0.3)$, we selected STAT4 and STAT5A for further experiments. Western blot was performed to measure the expression of STAT4 and STAT5A and their phosphorylation levels in MKN74 and MGC803 cells after treatment with $1.0 \mu \mathrm{g} / \mathrm{ml} 5-\mathrm{FU}$ for 6 and $12 \mathrm{~h}$. As shown in Figure 5E, 5-FU significantly increased STAT4, STAT5A, and their phosphorylation levels after treatment for $12 \mathrm{~h}$. Since the expression of STAT4 in gastric cancer cells is low or undetectable, we evaluated the correlation of STAT5A and PD-L1 in the further investigation. After STAT5A knockdown by siRNA, PD-L1 expression levels in MKN74 and MGC803 were downregulated (Figure 5F). Our previous study revealed that the miR-940/Cbl-b/STAT5A axis might regulate PD-L1 in gastric cancer cells (30). Given that Cbl-b could directly interact with STAT5A and increase the ubiquitination of STAT5A (30), we further examined whether the Cbl-b is involved in 5-FUinduced upregulation of STAT5A and PD-L1 in gastric cancer cells. Due to the higher expression of Cbl-b in MGC803, we silenced Cbl-b with siRNA followed by 5 -FU treatment. Western blot detected the expressions of Cbl-b, STAT5A, and PD-L1. The results showed that Cbl-b expression was decreased after 5-FU treatment. Meanwhile, silencing of Cbl-b increased 5FU-induced upregulation of p-STAT5 and PD-L1 (Figure 5G), indicating that 5-FU activated STAT5A and increased PDL1 expression through downregulation of Cbl-b. Given that miR-940 was found in the $3^{\prime}$-untranslated region (UTR) of Cbl-b transcript (30), we detected the expression of miR940 in MGC803. Quantitative RT-PCR analysis showed that the relative level of miR-940 was significantly increased in MGC803 cells following 5-FU treatment $(P<0.01$; Figure $5 \mathbf{H})$. Next, in order to investigate the effect of miR-940 on 5-FUinduced upregulation of PD-L1 expression, MGC803 cells were transfected with mimics of miR-940 or negative control followed by 5 -FU treatment. The results showed that transfected miR940 mimics further decreased 5-FU-induced Cbl-b expression, whereas p-STAT5 and PD-L1 were increased (Figure 5I). Taken together, these results indicated that 5-FU-induced upregulation of PD-L1 is mediated by miR-940/Cbl-b/STAT5A in gastric cancer cells.

\section{DISCUSSION}

Chemotherapy is the conventional treatment for advanced cancer but shows a limited survival advantage. Immunotherapy, which is characterized by immune checkpoint blockade (ICB), has changed the standard of care for a subgroup of patients and improved survival compared with previous criteria in several cancer types, even though limitations remain $(31,32)$. The efficacy of chemotherapy combined with ICB has been evaluated by several preclinical studies and clinical trials in the recent years. Combination therapies successfully provided survival benefit for patients in some cancer types, such as nonsmall-cell lung carcinoma (NSCLC), while they failed in other cancer types, such as gastric cancer (33-35). The KEYNOTE062 clinical study found that pembrolizumab plus chemotherapy as the first-line therapy did not show superior overall survival (OS) and progression-free survival (PFS) in advanced gastric 
A

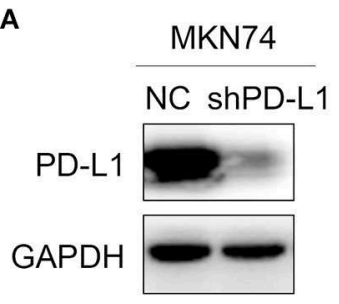

B

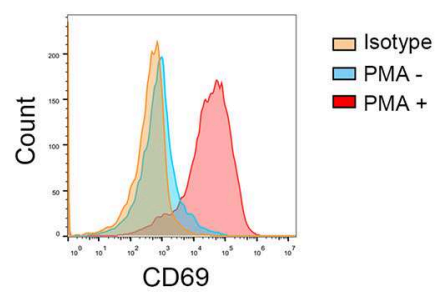

C
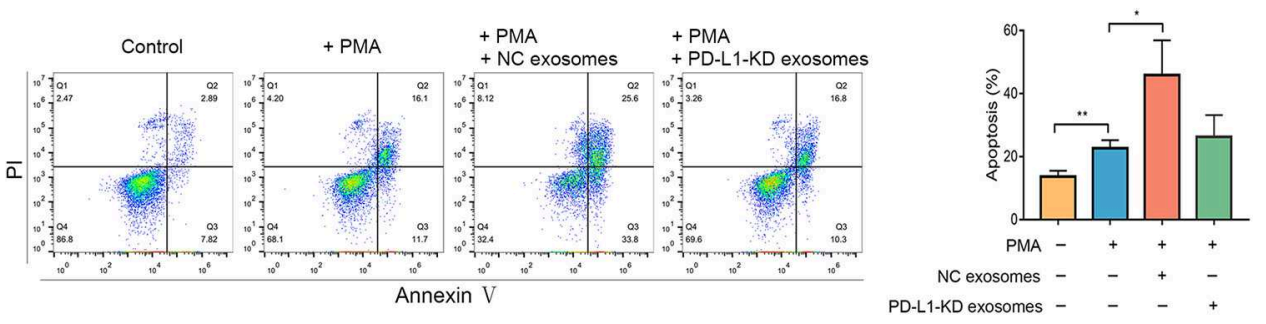

D

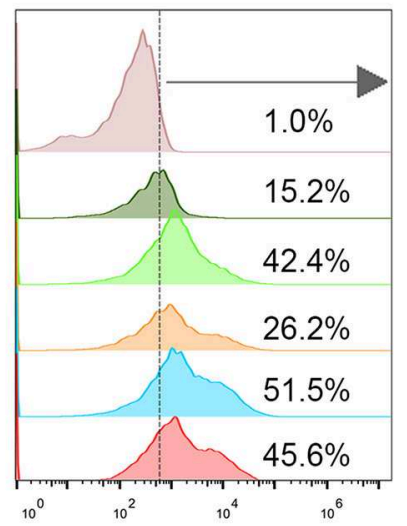

CD69 level on the membrane

E

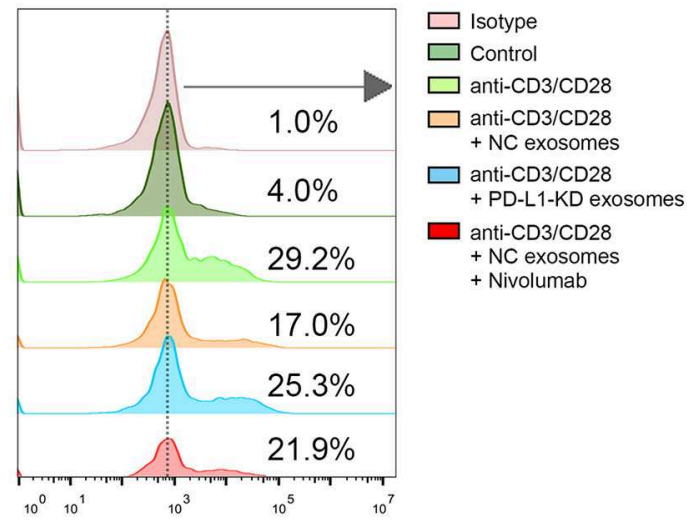

CD25 level on the membrane

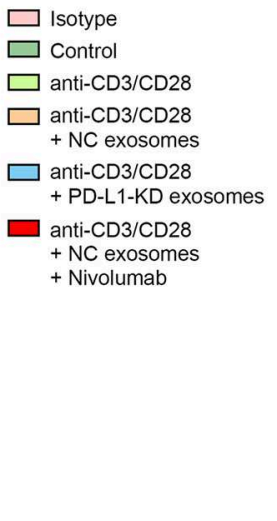

PD-L1-KD exosomes - $\quad-\quad+\quad+$

Nivolumab - $-\quad-\quad+$

FIGURE 4 | Exosomal programmed death-ligand 1 (PD-L1) induces Jurkat T cell apoptosis and suppresses T cell activation in peripheral blood mononuclear cells (PBMCs). (A) Western blot showed knockdown effect of PD-L1 in MKN74 cells. (B) Jurkat T cells were treated with 50 ng/ml phorbol 12-myristate 13-acetate (PMA) 
FIGURE 4 | for $24 \mathrm{~h}$, and activated Jurkat T cells were verified by high expression of CD69 on the surface using flow cytometry analysis. (C) Activated Jurkat T cells were coincubated with exosomes from PD-L1-NC and PD-L1-KD groups for 48 h, respectively. The apoptosis in activated Jurkat T cells was measured by flow cytometry analysis. (D,E) PBMCs were isolated from healthy human volunteers $(N=3)$. T cells in PBMCs were activated by human CD3/CD28T cell activator for $6 \mathrm{~h}$ at $37^{\circ} \mathrm{C}$ and then coincubated with exosomes from PD-L1-NC and PD-L1-KD groups for 48 h. Activated T cells in PBMC exposure to NC exosomes in the presence or absence of anti-PD-L1 antibody nivolumab for $16 \mathrm{~h}$. The activation markers CD69 and CD25 levels were analyzed by flow cytometry. Statistical analysis was performed by one-way ANOVA. $\left({ }^{*} P<0.05,{ }^{* *} P<0.01\right)$. Data were representative of three independent experiments.

cancer (36). However, the molecular mechanism underlying this combined treatment requires further elucidation. In the present study, our results indicated that 5-FU treatment could exert immunosuppressive effects through upregulation of PD-L1 expression in gastric cancer. This might be a reason that patients have not benefited from combination therapy.

Increasing studies have focused on the role of exosomal PDL1 in the recent years (37). Exosomes could transfer functional PD-L1 locally and distantly to suppress $\mathrm{T}$ cell activation and proliferation $(18,19)$. Previous study indicates that deletion of exosomal PD-L1 results in significantly decrease percentage of $\mathrm{Tim}^{+}$cells and increased Granzyme B $\mathrm{T}$ cells in mouse prostate cancer models (20). In addition, exosomal PD-L1 inhibits the proliferation of $\mathrm{CD}^{+} \mathrm{T}$ cells, as reflected by decreased expression levels of Ki-67 and Granzyme B, which promotes the progression of melanoma both in vivo and in vitro (19). Consistent with the suppressive effects of exosomal PD-L1 on T cell function, our study suggested that MKN74cell-derived exosomal PD-L1 induced apoptosis in Jurkat T cells and inhibited $\mathrm{T}$ cell activation in PBMCs, which could be attenuated by nivolumab. Few studies demonstrated that there is a significant correlation between high level of circulating exosomal PD-L1 with clinicopathological characters such as advanced tumor stage, disease progression, and lymph node involvement $(20,38)$. Our previous study has demonstrated that high exosomal PD-L1 was an independent prognostic factor, which was significantly associated with advanced tumor stage and poor overall survival (21). The study published in Nature in 2018 indicated that circulating exosome PD-L1 increased in clinical responders undergoing pembrolizumab (19). However, no studies reported the effects of chemotherapy on circulating exosomal PD-L1 in clinical responders and non-responders. Importantly, our results indicated that circulating exosomal PDL1 in gastric cancer patients increased significantly after two, four, and six cycles of fluoropyrimidine treatment, and the increase in circulating exosomal PD-L1 was more pronounced in non-responders than in responders. These findings suggest that upregulation of circulating exosomal PD-L1 might be a mechanism by which 5 -FU induced immunosuppression in gastric cancer.

Cytotoxic cytokines play an important role in promoting antitumor immunity. IFN- $\gamma$ is a critical cytokine secreted by activated $\mathrm{T}$ cells and plays crucial roles in promoting antitumor immune responses (39). TNF- $\alpha$ produced by various cell types, including lymphocytes and macrophages, is highly involved in the regulation of immune responses (40). IL-2 is termed as a key positive regulator of $\mathrm{CD} 4^{+} \mathrm{T}$ cell differentiation and function and can regulate the effector and memory responses of $\mathrm{CD}^{+}$ $\mathrm{T}$ cells (41). GM-CSF was reported to inhibit bladder cancer growth by reducing lymphangiogenesis and recruitment of M2 macrophages (42). Our results showed that several cytokines such as IFN- $\gamma$, TNF- $\alpha$, IL-2, IL-6, and GM-CSF were significantly reduced in the plasma of gastric cancer patients following repeated cycles of fluoropyrimidine treatment, especially after two cycles, suggesting that the functions of $\mathrm{T}$ cells and immune responses were impaired by fluoropyrimidine. Previous study has reported that exosomal PD-L1 derived from tumor cells inhibited the proliferation, cytokine production, and cytotoxicity of $\mathrm{CD}^{+} \mathrm{T}$ cells (19). However, results of our study showed that exosomal PD-L1 was not associated with the changes in immune cytokines after two or four cycles of fluoropyrimidine treatment. Considering that circulating $\mathrm{PD}-\mathrm{L}^{+}{ }^{+}$exosomes were secreted not only by tumor cells but also by antigen-presenting cells (APCs), it was unclear whether PD-L1 ${ }^{+}$exosomes produced by APC affect the immune response like exosomes derived from tumor cells (38). In addition to the aforementioned findings, our data showed that the absolute counts of $\mathrm{CD} 4^{+}$and $\mathrm{CD} 8^{+} \mathrm{T}$ cells decreased following fluoropyrimidine treatment, although the change was not statistically significant. Previous study has reported similar results as the $\mathrm{CD}^{+}$and $\mathrm{CD} 8^{+} \mathrm{T}$ cell amounts could recover quickly after chemotherapy, with mean $\mathrm{CD}^{+} \mathrm{T}$ cell amounts returning to baseline more quickly than $\mathrm{CD} 4^{+}$ $\mathrm{T}$ cell levels (43). Consistent with findings in CT26 tumorbearing mouse model (44), this study with clinical blood samples suggested that inhibition of key cytokines production might be a second mechanism by which 5-FU induced immunosuppression in gastric cancer.

Various regulatory mechanisms of PD-L1 expression have been studied, mainly including (1) gene alteration at the PD-L1 locus, (2) control of PD-L1 gene expression through inflammatory signaling, (3) aberrant oncogenic signaling influences PD-L1 expression, (4) miRNA-mediated PD-L1 mRNA regulation, and (5) $\mathrm{PD}-\mathrm{L} 1$ regulation at protein level (45-50). However, few studies have reported the mechanism of PD-L1 expression by 5 -FU induction. It was reported that Folfox (5-FU plus oxaliplatin) increases PD-L1 levels by IFN- $\gamma$ secreted by Folfox-induced $\mathrm{CD}^{+}{ }^{+} \mathrm{T}$ cells (51). In the present study, our results suggest that 5-FU-induced upregulation of PD-L1 might be mediated by miR-940/Cbl-b/STAT5A axis in gastric cancer cells.

In summary, we demonstrated that 5-FU upregulated gastric cancer cell-derived exosomal PD-L1 in a dose- and timedependent manner. Fluorouracil chemotherapy might exert immunosuppressive effects by upregulating exosomal PD-L1, accompanied by a decrease in cytokines in gastric cancer patients. Exosomal PD-L1 derived from gastric cancer cells induced apoptosis of Jurkat $\mathrm{T}$ cells and inhibited $\mathrm{T}$ cells activation in PBMCs. Additionally, our data suggested that 5-FU 

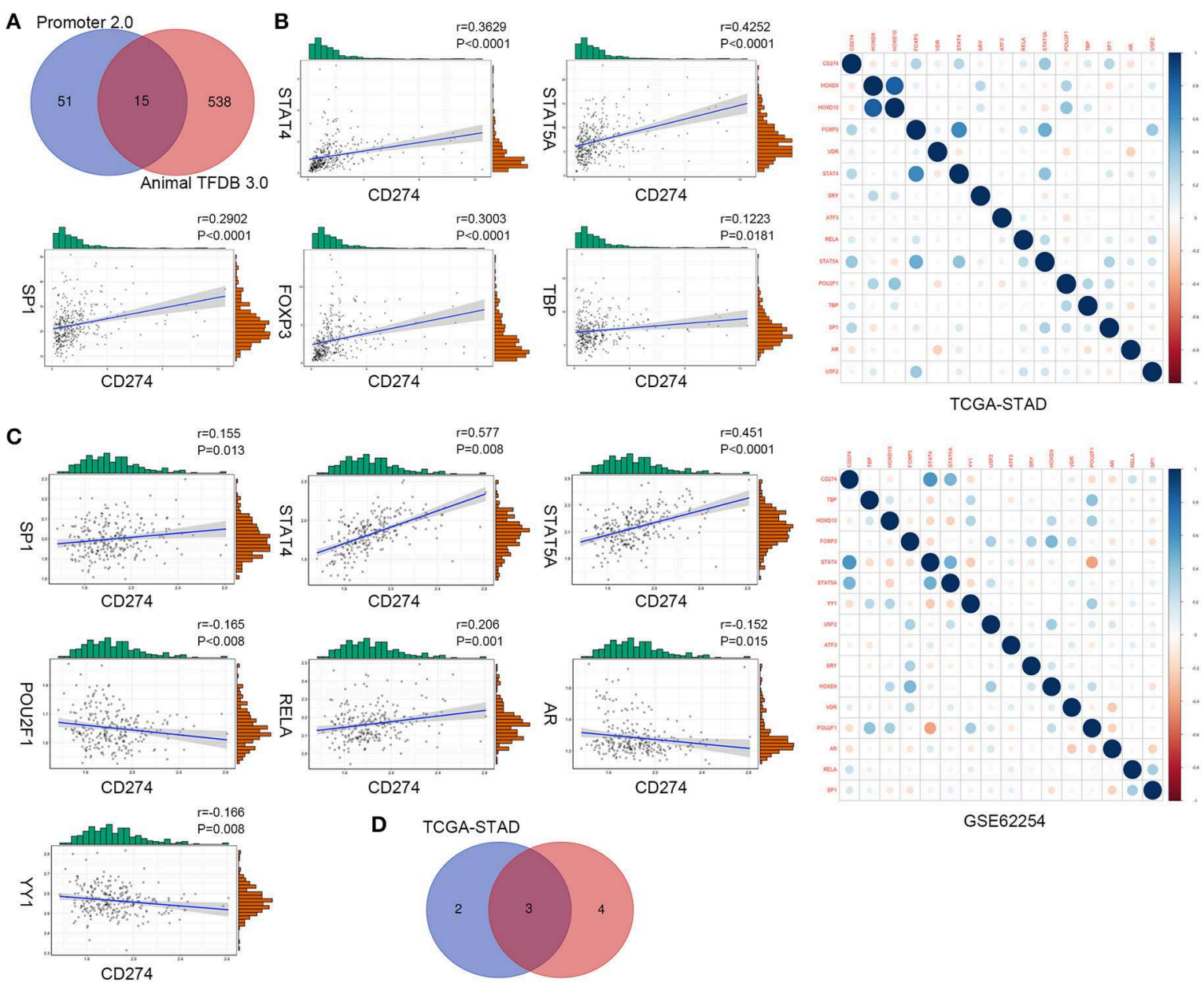

D TCGA-STAD

GSE62254

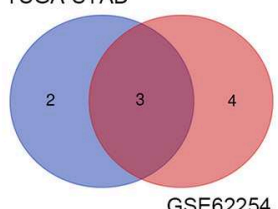

E

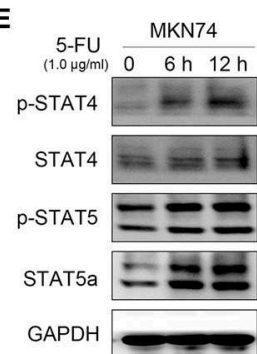

G

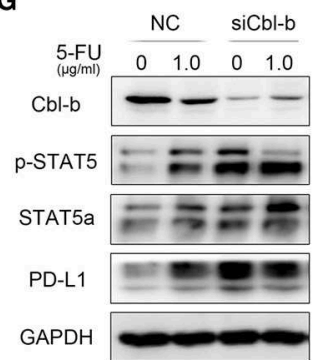

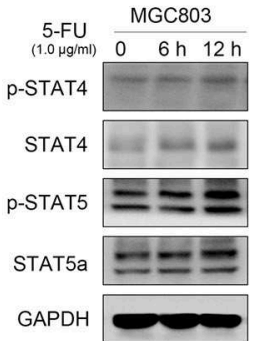

H

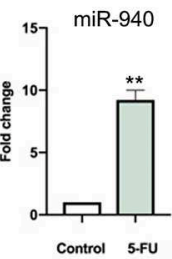

$\mathbf{F}$

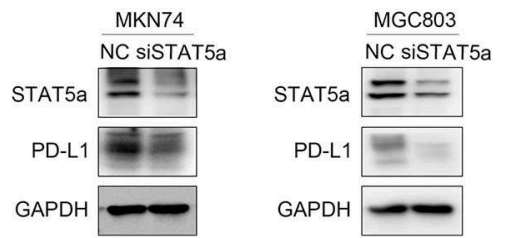

I

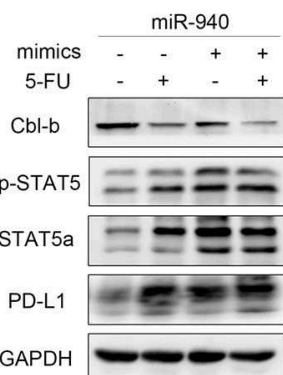

FIGURE 5 | MiR-940/Cbl-b/signal transducer and activator of transcription 5A (STAT5A) are involved in 5-fluorouracil (5-FU)-induced upregulation of programmed death-ligand 1 (PD-L1) in gastric cancer cells. (A) Online database Promoter 2.0 Prediction Server and Animal TFDB 3.0 predicted transcriptional factors of PD-L1. 
FIGURE 5 | (B-D) Correlation analysis of predicted transcriptional factors with PD-L1 using the gene expression profiles of TCGA-STAD database and GSE62254. (E) MKN74 and MGC803 cells were treated with $1.0 \mu \mathrm{g} / \mathrm{ml} 5$-FU for 6 or $12 \mathrm{~h}$; the phosphorylation of signal transducer and activator of transcription 4 (STAT4) and STAT5A were analyzed by Western blot. (F) After transient knockdown of STAT5A by using small interfering RNA (siRNA) for 72 h, STAT5A and PD-L1 levels were detected by Western blot analysis, and glyceraldehyde 3-phosphate dehydrogenase (GAPDH) was used as internal control. Data were representative of three independent experiments. (G) After transient knockdown of Cbl-b by using siRNA for $72 \mathrm{~h}$ and treated MGC803 with $1.0 \mu \mathrm{g} / \mathrm{ml} 5$-FU for $72 \mathrm{~h}$, simultaneously, Cbl-b, p-STAT5, STAT5A, and PD-L1 levels were detected by Western blot analysis. (H) The expression of miR-940 in MGC803 treated with or without $1.0 \mu \mathrm{g} / \mathrm{ml} 5$-FU for $72 \mathrm{~h}$ was analyzed. ${ }^{\star \star} P<0.01$. (I) MGC803 was cotransfected with $40 \mathrm{nmol}$ miR-940 mimics and treated with $1.0 \mu \mathrm{g} / \mathrm{ml} 5$-FU for $72 \mathrm{~h}$. Western blot analysis the expression of Cbl-b, p-STAT5, STAT5A, and PD-L1 in MGC803.

upregulated PD-L1 expression through miR-940/Cbl-b/STAT5A axis in gastric cancer cells. These results provide new insights into immunosuppressive effects of 5-FU. However, this study was limited due to the lack of a mouse model to validate the relationship between exosome PD-L1 and tumor immunity in vivo and whether inhibiting exosomal PD-L1 can improve immune activation targeting GC.

\section{DATA AVAILABILITY STATEMENT}

Our experimental data are unpublished data in the past and do not include data from other authors or other organizations. Part of the data come from the public free-charged database. The source of database has been added to the article.

\section{ETHICS STATEMENT}

The study was reviewed and approved by the Human Ethics Review Committee of the First Hospital of China Medical University, and all researches were conducted in accordance with ethical principles. All patients agree to participate in our study.

\section{AUTHOR CONTRIBUTIONS}

MZ and YF performed the experiments, analyzed the data, and wrote the manuscript. XC designed experiments and revised the manuscript. XQ and YL supervised the studies, obtained funding, and also revised the paper. KH, CZ, TW, CL, SW, and YC performed experiments. All authors read and approved the final manuscript.

\section{REFERENCES}

1. Bray F, Ferlay J, Soerjomataram I, Siegel RL, Torre LA, Jemal A. Global cancer statistics 2018: GLOBOCAN estimates of incidence and mortality worldwide for 36 cancers in 185 countries. Ca: Cancer J Clin. (2018) 68:394424. doi: $10.3322 /$ caac. 21492

2. Shah MA. Update on metastatic gastric and esophageal cancers. J Clin Oncol. (2015) 33:1760-9. doi: 10.1200/JCO.2014.60.1799

3. Zitvogel L, Apetoh L, Ghiringhelli F, Kroemer G. Immunological aspects of cancer chemotherapy. Nat Rev Immunol. (2008) 8:59-73. doi: 10.1038/nri2216

4. Mathios D, Kim JE, Mangraviti A, Phallen J, Park CK, Jackson CM, et al. Anti-PD-1 antitumor immunity is enhanced by local and abrogated by systemic chemotherapy in GBM. Sci Translat Med. (2016) 8:370ra180. doi: $10.1126 /$ scitranslmed.aag 2942

5. Ilson DH. Advances in the treatment of gastric cancer. Curr Opinion Gastroenterol. (2018) 34:465-468. doi: 10.1097/MOG.000000000 0000475

\section{FUNDING}

This research was supported by the National Key Research and Development Program of China (No. 2017YFC1308900), National Science and Technology Major Project of the Ministry of Science and Technology of China (No. 2017ZX09304025), National Natural Science Foundation of China (No. 81602098), National Natural Science Foundation of China (No. 31770963), The Key Research and Development Program of Liaoning Province (2018225060), Science and Technology Plan Project of Liaoning Province (No.2016007010), National Natural Science Foundation of China (No. 81803092), and Science and Technology Plan Project of Liaoning Province (No.2013225585).

\section{SUPPLEMENTARY MATERIAL}

The Supplementary Material for this article can be found online at: https://www.frontiersin.org/articles/10.3389/fonc. 2020.00492/full\#supplementary-material

Figure S1 | (A) Plot of circulating exosomal PD-L1 levels in gastric cancer patients at baseline and after six cycles of fluoropyrimidine chemotherapy treatment $(N=$ 3). (B) The levels of IFN- $\gamma$, TNF- $\alpha$, IL-2, IL-6, and GM-CSF in plasma were analyzed by multiplex bead assay at baseline and after four and six cycles of fluoropyrimidine chemotherapy. (C) Plot of changes of IFN- $\gamma$, TNF- $\alpha$, IL-2, IL-6, and GM-CSF levels in plasma in responders and non-responders groups. The two-tailed paired $t$-test was used in statistical analysis where appropriate to evaluate the statistical significance $\left({ }^{*} P<0.05,{ }^{* *} P<0.01\right)$.

Tables S1 and S2 | Correlation between the changes of exosomal PD-L1 and eight immune cytokines in the clinical blood samples after two and four cycles of treatment.

6. Longley DB, Harkin DP, Johnston PG. 5-fluorouracil: mechanisms of action and clinical strategies. Nat Rev Cancer. (2003) 3:330-8. doi: 10.1038/nrc1074

7. Vincent J, Mignot G, Chalmin F, Ladoire S, Bruchard M, Chevriaux A, et al. 5-Fluorouracil selectively kills tumor-associated myeloid-derived suppressor cells resulting in enhanced T cell-dependent antitumor immunity. Cancer Res. (2010) 70:3052-61. doi: 10.1158/0008-5472.CAN-09-3690

8. Galluzzi L, Buque A, Kepp O, Zitvogel L, Kroemer G. Immunological effects of conventional chemotherapy and targeted anticancer agents. Cancer cell. (2015) 28:690-714. doi: 10.1016/j.ccell.2015.10.012

9. Deng C, Zhang Q, Jia M, Zhao J, Sun X, Gong T, et al. Tumors and their microenvironment dual-targeting chemotherapy with local immune adjuvant therapy for effective antitumor immunity against breast cancer. Adv Sci. (2019) 6:1801868. doi: 10.1002/advs.201801868

10. Ding ZC, Munn DH, Zhou G. Chemotherapy-induced myeloid suppressor cells and antitumor immunity: the Janus face of chemotherapy in immunomodulation. Oncoimmunology. (2014) 3:e954471. doi: 10.4161/21624011.2014.954471 
11. Zhang P, Su DM, Liang M, Fu J. Chemopreventive agents induce programmed death-1-ligand 1 (PD-L1) surface expression in breast cancer cells and promote PD-L1-mediated T cell apoptosis. Mol Immunol. (2008) 45:14706. doi: 10.1016/j.molimm.2007.08.013

12. Yang M, Liu P, Wang K, Glorieux C, Hu Y, Wen S, et al. Chemotherapy induces tumor immune evasion by upregulation of programmed cell death ligand 1 expression in bone marrow stromal cells. Mol Oncol. (2017) 11:35872. doi: 10.1002/1878-0261.12032

13. Keklikoglou I, Cianciaruso C, Guc E, Squadrito ML, Spring LM, Tazzyman S, et al. Chemotherapy elicits pro-metastatic extracellular vesicles in breast cancer models. Nat Cell Biol. (2019) 21:190-202. doi: 10.1038/s41556-018-0256-3

14. Tran L, Allen CT, Xiao R, Moore E, Davis R, Park SJ, et al. Cisplatin alters antitumor immunity and synergizes with PD-1/PD-L1 inhibition in head and neck squamous cell carcinoma. Cancer Immunol Res. (2017) 5:114151. doi: 10.1158/2326-6066.CIR-17-0235

15. Taylor DD, Gercel-Taylor C. Exosomes/microvesicles: mediators of cancerassociated immunosuppressive microenvironments. Semin Immunopathol. (2011) 33:441-54. doi: 10.1007/s00281-010-0234-8

16. Shah R, Patel T, Freedman JE. Circulating extracellular vesicles in human disease. N Engl J Med. (2018) 379:2180-1. doi: 10.1056/NEJMc1813170

17. Jeppesen DK, Fenix AM, Franklin JL, Higginbotham JN, Zhang Q, Zimmerman LJ, et al. Reassessment of exosome composition. Cell. (2019) 177:428-45.e18. doi: 10.1016/j.cell.2019.02.029

18. Yang Y, Li CW, Chan LC, Wei Y, Hsu JM, Xia W, et al. Exosomal PD-L1 harbors active defense function to suppress $T$ cell killing of breast cancer cells and promote tumor growth. Cell Res. (2018) 28:8624. doi: 10.1038/s41422-018-0060-4

19. Chen G, Huang AC, Zhang W, Zhang G, Wu M, Xu W, et al. Exosomal PD-L1 contributes to immunosuppression and is associated with anti-PD-1 response. Nature. (2018) 560:382-6. doi: 10.1038/s41586-018-0392-8

20. Poggio M, Hu T, Pai CC, Chu B, Belair CD, Chang A, et al. Suppression of exosomal PD-L1 induces systemic anti-tumor immunity and memory. Cell. (2019) 177:414-27.e13. doi: 10.1016/j.cell.2019.02.016

21. Fan Y, Che X, Qu J, Hou K, Wen T, Li Z, et al. Exosomal PD-L1 retains immunosuppressive activity and is associated with gastric cancer prognosis. Annal Surg Oncol. (2019) 26:3745-55. doi: 10.1245/s10434-019-07431-7

22. Deng G, Qu J, Zhang Y, Che X, Cheng Y, Fan Y, et al. Gastric cancer-derived exosomes promote peritoneal metastasis by destroying the mesothelial barrier. FEBS Lett. (2017) 591:2167-79. doi: 10.1002/1873-3468.12722

23. Shi $\mathrm{X}$, Zheng $\mathrm{C}$, Li C, Hou K, Wang X, Yang Z, et al. 4-Phenybutyric acid promotes gastric cancer cell migration via histone deacetylase inhibition-mediated HER3/HER4 up-regulation. Cell Biol Int. (2018) 42:5362. doi: 10.1002/cbin.10866

24. L. Van Der Kraak, Goel G, Ramanan K, Kaltenmeier C, Zhang L, Normolle DP, et al. 5-Fluorouracil upregulates cell surface B7-H1 (PDL1) expression in gastrointestinal cancers. J Immunother Cancer. (2016) 4:65. doi: 10.1186/s40425-016-0163-8

25. Gong W, Song Q, Lu X, Gong W, Zhao J, Min P, et al. Paclitaxel induced B7H1 expression in cancer cells via the MAPK pathway. J Chemother. (2011) 23:295-9. doi: 10.1179/joc.2011.23.5.295

26. Chen Y, Wang Q, Shi B, Xu P, Hu Z, Bai L, et al. Development of a sandwich ELISA for evaluating soluble PD-L1 (CD274) in human sera of different ages as well as supernatants of PD-L1+ cell lines. Cytokine. (2011) 56:231-8. doi: 10.1016/j.cyto.2011.06.004

27. Zhou J, Mahoney KM, Giobbie-Hurder A, Zhao F, Lee S, Liao $\mathrm{X}$, et al. Soluble PD-L1 as a biomarker in malignant melanoma treated with checkpoint blockade. Cancer Immunol Res. (2017) 5:480-92. doi: 10.1158/2326-6066.CIR-16-0329

28. Dai S, Jia R, Zhang $\mathrm{X}$, Fang Q, Huang L. The PD-1/PDLs pathway and autoimmune diseases. Cell Immunol. (2014) 290:72-9. doi: 10.1016/j.cellimm.2014.05.006

29. Ricklefs FL, Alayo Q, Krenzlin H, Mahmoud AB, Speranza MC, Nakashima $\mathrm{H}$, et al. Immune evasion mediated by PD-L1 on glioblastoma-derived extracellular vesicles. Sci Adv. (2018) 4:eaar2766. doi: 10.1126/sciadv.aar2766

30. Fan Y, Che X, Hou K, Zhang M, Wen T, Qu X, et al. MiR-940 promotes the proliferation and migration of gastric cancer cells through up-regulation of programmed death ligand-1 expression. Exp Cell Res. (2018) 373:1807. doi: 10.1016/j.yexcr.2018.10.011

31. Fuchs CS, Doi T, Jang RW, Muro K, Satoh T, Machado M, et al. Safety and efficacy of pembrolizumab monotherapy in patients with previously treated advanced gastric and gastroesophageal junction cancer: Phase 2 clinical KEYNOTE-059 trial. JAMA Oncol. (2018) 4:e180013. doi: 10.1001/jamaoncol.2018.0013

32. Garon EB, Rizvi NA, Hui R, Leighl N, Balmanoukian AS, Eder JP, et al. Pembrolizumab for the treatment of non-small-cell lung cancer. $N$ Engl J Med. (2015) 372:2018-28. doi: 10.1056/NEJMoa1501824

33. Paz-Ares L, Luft A, Vicente D, Tafreshi A, Gumus M, Mazieres J, et al. Pembrolizumab plus chemotherapy for squamous non-small-cell lung cancer. N Engl J Med. (2018) 379:2040-51. doi: 10.1056/NEJMoa1810865

34. Gandhi L, Rodriguez-Abreu D, Gadgeel S, Esteban E, Felip E, De Angelis F, et al. Pembrolizumab plus chemotherapy in metastatic non-small-cell lung cancer. N Engl J Med. (2018) 378:2078-92. doi: 10.1056/NEJMoa1801005

35. Cui S. Immunogenic chemotherapy sensitizes renal cancer to immune checkpoint blockade therapy in preclinical models. Med Sci Monitor. (2017) 23:3360-6. doi: 10.12659/MSM.902426

36. Tabernero, Van Cutsem E, Bang Y, Fuchs C, Wyrwicz L, Lee K, et al. Pembrolizumab with or without chemotherapy versus chemotherapy for firstline treatment of advanced gastric or gastroesophageal junction (G/GEJ) adenocarcinoma: the Phase 3 KEYNOTE-062 study. Annal Oncol. (2019) 30(Suppl. 4):iv152-3. doi: 10.1093/annonc/mdz183.001

37. Daassi D, Mahoney KM, Freeman GJ. The importance of exosomal PDL1 in tumour immune evasion. Nat Rev Immunol. (2020) 20:20915. doi: 10.1038/s41577-019-0264-y

38. Theodoraki MN, Yerneni SS, Hoffmann TK, Gooding WE, Whiteside TL. Clinical significance of PD-L1(+) exosomes in plasma of head and neck cancer patients. Clin Cancer Res. (2018) 24:896-905. doi: 10.1158/1078-0432.CCR-17-2664

39. Ikeda H, Old LJ, Schreiber RD. The roles of IFN gamma in protection against tumor development and cancer immunoediting. Cytokine Growth Fact Rev. (2002) 13:95-109. doi: 10.1016/S1359-6101(01)00038-7

40. Sedger LM, McDermott MF. TNF and TNF-receptors: from mediators of cell death and inflammation to therapeutic giants - past, present and future. Cytokine Growth Fact Rev. (2014) 25:453-72. doi: 10.1016/j.cytogfr.2014.07.016

41. Spolski R, Li P, Leonard WJ. Biology and regulation of IL-2: from molecular mechanisms to human therapy. Nat Rev Immunol. (2018) 18:64859. doi: 10.1038/s41577-018-0046-y

42. Hori S, Miyake M, Onishi S, Morizawa Y, Nakai Y, Tatsumi Y, et al. Evaluation of pro and antitumor effects induced by three colonystimulating factors, GCSF, GMCSF and MCSF, in bladder cancer cells: Is GCSF a friend of bladder cancer cells? Int J Oncol. (2019) 54:2237-49. doi: 10.3892/ijo.2019.4772

43. Mackall CL, Fleisher TA, Brown MR, Andrich MP, Chen CC, Feuerstein IM, et al. Distinctions between CD8+ and CD4+ T-cell regenerative pathways result in prolonged $\mathrm{T}$-cell subset imbalance after intensive chemotherapy. Blood. (1997) 89:3700-7. doi: 10.1182/blood.V89.10.3700.3700_3700 -3707

44. Wu Y, Deng Z, Wang H, Ma W, Zhou C, Zhang S. Repeated cycles of 5-fluorouracil chemotherapy impaired anti-tumor functions of cytotoxic $\mathrm{T}$ cells in a CT26 tumor-bearing mouse model. BMC Immunol. (2016) 17:29. doi: 10.1186/s12865-016-0167-7

45. Sun C, Mezzadra R, Schumacher TN. Regulation and Function of the PD-L1 checkpoint. Immunity. (2018) 48:434-52. doi: 10.1016/j.immuni.2018.03.014

46. Liu J, Hamrouni A, Wolowiec D, Coiteux V, Kuliczkowski K, Hetuin D, et al. Plasma cells from multiple myeloma patients express B7-H1 (PDL1) and increase expression after stimulation with IFN-\{gamma $\}$ and TLR ligands via a MyD88-, TRAF6-, and MEK-dependent pathway. Blood. (2007) 110:296-304. doi: 10.1182/blood-2006-10-051482

47. Platanias LC. Mechanisms of type-I- and type-II-interferon-mediated signalling. Nat Rev Immunol. (2005) 5:375-86. doi: 10.1038/nri1604

48. Lee SJ, Jang BC, Lee SW, Yang YI, Suh SI, Park YM, et al. Interferon regulatory factor- 1 is prerequisite to the constitutive expression and IFNgamma-induced upregulation of B7-H1 (CD274). FEBS Lett. (2006) 580:75562. doi: 10.1016/j.febslet.2005.12.093 
49. Parsa AT, Waldron JS, Panner A, Crane CA, Parney IF, Barry JJ, et al. Loss of tumor suppressor PTEN function increases B7-H1 expression and immunoresistance in glioma. Nat Med. (2007) 13:84-8. doi: 10.1038/nm1517

50. Atefi M, Avramis E, Lassen A, Wong DJ, Robert L, Foulad D, et al. Effects of MAPK and PI3K pathways on PD-L1 expression in melanoma. Clin Cancer Res. (2014) 20:3446-57. doi: 10.1158/1078-0432.CCR13-2797

51. Dosset M, Vargas TR, Lagrange A, Boidot R, Vegran F, Roussey A, et al. PD-1/PD-L1 pathway: an adaptive immune resistance mechanism to immunogenic chemotherapy in colorectal cancer. Oncoimmunology. (2018) 7:e1433981. doi: 10.1080/2162402X.2018.1433981
Conflict of Interest: The authors declare that the research was conducted in the absence of any commercial or financial relationships that could be construed as a potential conflict of interest.

Copyright $\odot 2020$ Zhang, Fan, Che, Hou, Zhang, Li, Wen, Wang, Cheng, Liu and Qu. This is an open-access article distributed under the terms of the Creative Commons Attribution License (CC BY). The use, distribution or reproduction in other forums is permitted, provided the original author(s) and the copyright owner(s) are credited and that the original publication in this journal is cited, in accordance with accepted academic practice. No use, distribution or reproduction is permitted which does not comply with these terms. 